\title{
Influence of Effective Angle of Attack Oscillation Amplitude on Force Generation by Pitching-Plunging Flat Plates
}

\author{
Victor Pinedo ${ }^{1}$, and Luis Bernal ${ }^{2}$ \\ University of Michigan, Ann Arbor, MI
}

\begin{abstract}
An experimental study exploring the effective angle of attack oscillation amplitude on pitching and plunging flat plates is presented, as an extension of previous studies on pitching-plunging flat plate airfoils with harmonic effective angle of attack history. We consider the effect of reduced frequency and plunge motion amplitude. Two sets of test cases are studied: a shallow dynamic stall $\left(-6^{\circ}<\alpha_{\text {eff }}<22^{\circ}\right)$, and a deep dynamic stall $\left(-2.35^{\circ}<\alpha_{\text {eff }}\right.$ $<\mathbf{1 3 . 6 5}^{\circ}$ ). Force measurements are shown, and PIV data is presented for the shallow stall cases. Results show that for the shallow stall cases, the LEVs are significantly smaller in size than for the deep stall cases reported in literature. The force measurement results show significant impact of unsteady effects on the force time history, particularly for the more aggressive motions. Shallow stall cases delay the lift force history by almost a quarter cycle. In addition, significant lift generation is observed in the shallow cases given the narrow range of $\alpha_{\text {eff }}$ used. Non-circulatory effects or rotation rate effects appear to be more dominant than LEV in force generation for the shallow stall cases.
\end{abstract}

\section{Introduction}

The present work analyzes the effect of the effective angle of attack $\left(\alpha_{\text {eff }}\right)$ oscillation amplitude on the aerodynamics of flat plate airfoils undergoing pitching and plunging kinematics at low Reynolds numbers ( $\operatorname{Re}$ 5000). This work is an extension of previous studies on pitching-plunging flat plate airfoils [1-5] and attempts to shed light on the unsteady flow development and force generation dependence on $\alpha_{\text {eff }}$ history, keeping all other parameters constant. We consider the same reduced frequency $(k)$ and non-dimensional amplitude $\left(h_{0}\right)$ parameter space as Baik et al. [1], who found that the Strouhal number (St) is the main parameter determining unsteady force generation. Baik et al. also characterized the formation, evolution, and detachment of leading edge vortices, which contribute to force generation and results in force coefficients significantly larger than predicted by potential flow theory. They found that the flow evolution is primarily controlled by reduced frequency. Baik et al. [1] investigated a deep dynamic stall kinematics $\left(-6^{\circ}<\alpha_{\mathrm{eff}}<22^{\circ}\right)$ in which the maximum effective angle of attack was well beyond static stall. Motivated by $\mathrm{Ol}$ et al. [5], in the present study we consider also a shallow stall kinematics $\left(-2.35^{\circ}<\alpha_{\text {eff }}\right.$ $<13.65^{\circ}$ ) in which the maximum effective angle of attack is slightly above static stall for the same reduced frequency and non-dimensional plunge amplitude as in the deep stall case. At the smaller effective angle of attack, leading edge vortex strength should be smaller, and its impact on force generation reduced. At the same time the smaller effective angle of attack results in more aggressive pitching motion for the same plunge motion amplitude, which may impact force generation. As in Baik et al. the St values chosen range from 0.16 to 0.32 , which is relevant to biological fliers [6] and is believed to be optimal for thrust production [7]. Experimental work is done in a low turbulence water channel using an infinite aspect ratio flat plate wing, and direct force measurements are performed to analyze the time history effects of the different kinematics. Particle Image Velocimetry (PIV) measurements are also performed on the cases of shallow dynamic stall, to analyze the force generation and determine the role of LEV development in these flows.

This work is motivated by recent interest in the aerodynamics of flapping wing Micro Air Vehicles, and their various applications and flight regimes [8,9]. Airfoils undergoing periodic pitching and plunging motion have been studied [10-12] in order to understand the effect of vortical structures in the flow. Leading edge vortices have been found to develop in large amplitude flapping wing motions at low and moderate Reynolds numbers, and are believed

\footnotetext{
${ }^{1}$ Graduate Student, Department of Aerospace Engineering, University of Michigan, vpinedo@umich.edu, AIAA student member

${ }^{2}$ Associate Professor, Department of Aerospace Engineering, University of Michigan, lpb@umich.edu, AIAA member
} 
to be the main mechanism for lift generation of biological fliers [13-15]. It has been found that for 2D thin flat plate airfoils, Reynolds number effects are small [3,4] at Re 3-6x10 ${ }^{4}$. In addition, in the present study we consider only plunge motions producing a sinusoidal effective angle of attack profile which for the present large values of St numbers help avoid non-harmonic $\alpha_{\text {eff }}$ effects and has been shown to provide optimal thrust [16-18].

\section{Experimental Setup}

\section{A. Laboratory Setup}

The experiments are performed at the University of Michigan's low-turbulence water channel. The cross section of the channel is $2 \mathrm{ft} \times 2 \mathrm{ft}$, and the free-stream velocity ranges from $6 \mathrm{~cm} / \mathrm{s}$ to $40 \mathrm{~cm} / \mathrm{s}$. The turbulence intensity has been measured to be $\sim 1 \%$ [1]. The free-stream velocity (in $\mathrm{cm} / \mathrm{s}$ ) is a function of the motor speed $(M)$ and the water height in the test section $(H)$. The free-stream velocity was calibrated using PIV velocity measurements at the center of the test section for steady operation and found to be given the correlation,

$$
U_{\infty}=[2.43 \times M-0.082] \times \frac{H_{0}}{H}
$$

where, $H_{0}=22$ ". The water height and the motor speed for all the present experiments was $H=23.25$ " and $M=2.9$, respectively, which give a nominal free-stream speed $U_{\infty}=6.6 \mathrm{~cm} / \mathrm{s}$, and Reynolds number $R e \approx 5000$, consistent with Baik's work [1].

The pitch and plunge motions are controlled by two stepper motors. A linear traverse generates the plunge motion, and a rotary stage generates the pitch motion. The resolution of the linear traverse is 1000 steps per inch, and that of the rotary stage is 80 steps per degree. A synchronization between the pitch and plunge motions is enforced using synchronization signals in the controllers so that the motions are always in phase. For all tests, the kinematics have been optimized to produce the least amount of holding time and the closest match between the periods for the pitch and plunge motions. The measured periods are within $1.5 \%$ of the nominal periods.

The model is a two-dimensional (infinite aspect ratio) flat plate with rounded corners and the following dimensions: chord, $c=7.62 \mathrm{~cm}$, thickness, $t h=0.48 \mathrm{~cm}$, wingspan, $b=57.15 \mathrm{~cm}$. For the force measurements, the model is mounted on an L-bracket attached to a six-component force sensor, which in turn is mounted on a bracket attached to the rotary stage and linear traverse. For all tests, the bottom edge of the plate is about $1 \mathrm{~mm}$ from the bottom of the water channel. Figure 1 shows the mounting of the plate, the stepper motors, and the force sensor.
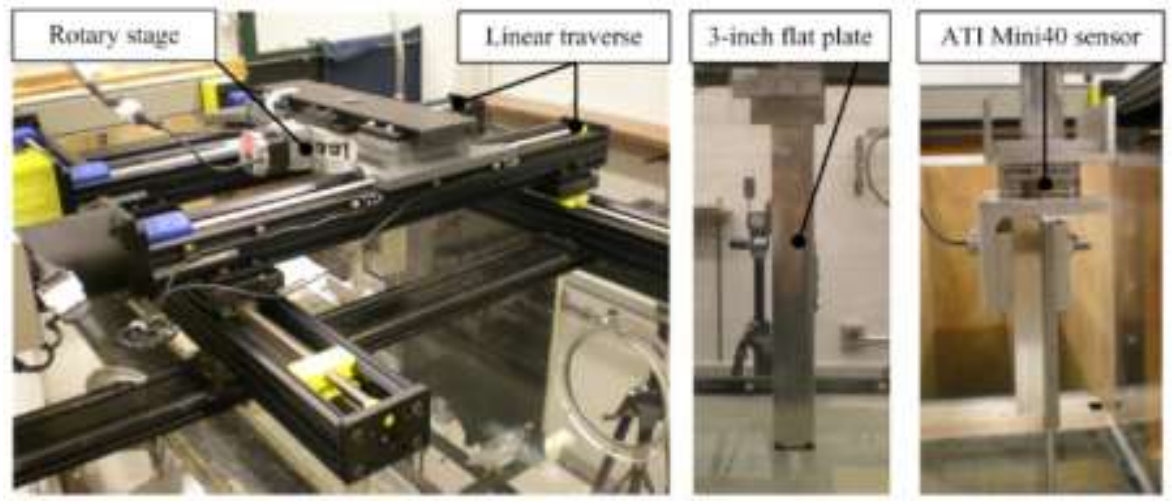

Figure 1. Experimental setup of flat plate, force sensor, and motors

The force sensor used is a 6-component ATI Industrial Automation Mini40 transducer. It has a nominal force resolution of $1 / 50 \mathrm{~N}$, and a nominal torque resolution of $1 / 2000 \mathrm{~N}-\mathrm{m}$. The sensor data is digitized with a National Instrument PCI-6625 DAQ card. 


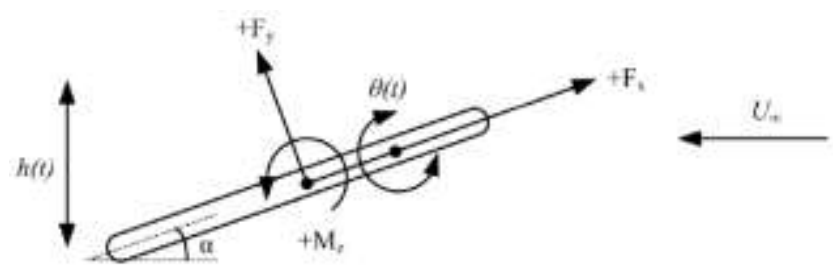

\section{Figure 2. Orientation of forces and pivot point with respect to incoming flow}

The force sensor location is at the half-chord, to prevent excessive torque loads on the transducer. The pivot point location is at the quarter-chord. The orientation of the force sensor and pivot point on the flat plate with respect to the flow is shown in Figure 2. From these equations and the orientation of the sensor, the following equations describe the lift and drag, as measured by the sensor:

$$
\begin{gathered}
L=F_{x} \sin \alpha+F_{y} \cos \alpha \\
D=-F_{x} \cos \alpha+F_{y} \sin \alpha \\
c_{L}=\frac{L}{0.5 \rho c b U_{\infty}{ }^{2}} \\
c_{D}=\frac{D}{0.5 \rho c b U_{\infty}{ }^{2}}
\end{gathered}
$$

Tests were performed in water at $U_{\infty}=6.6 \mathrm{~cm} / \mathrm{s}$ and in air (tare test). The latter is done so it can be subtracted from the water force data, in order to remove inertial loads on the model and only retain the hydrodynamic forces. For each case, 6 data sets were taken, and each data set includes 31 cycles, out of which the first 5 and the last one are discarded to remove start-up and stopping transients.

The Particle Image Velocimetry (PIV) in two dimensions is obtained by using two lasers, light sheet forming optics, a high-resolution camera, control electronics, and a computer system. The laser used is a double-pulsed NdYAG laser, and the laser wavelength is $\lambda=532 \mathrm{~nm}$, seen as green light. The camera used is a two-frame highresolution CCD digital camera, PCO 4000, with a resolution of 4008x2672 pixels and low noise (11e- rms @ $8 \mathrm{MHz}$ ). The light sheet forming optics consists of 1 spherical lens, 1 cylindrical lens, and reflecting mirrors. The spherical lens is used to produce a relatively small thickness in one direction while the cylindrical lens is used for beam divergence in the orthogonal direction, which creates the light sheet. Since the sheet is parallel to the cross flow, it is expected that the cross-plane velocity will be minimal. The two laser sheets are aligned carefully, and this alignment is checked by taking a picture of a ruler and ensuring that marker points are in the same pixel position. Figures $3 \& 4$ show a schematic of the experimental setup.

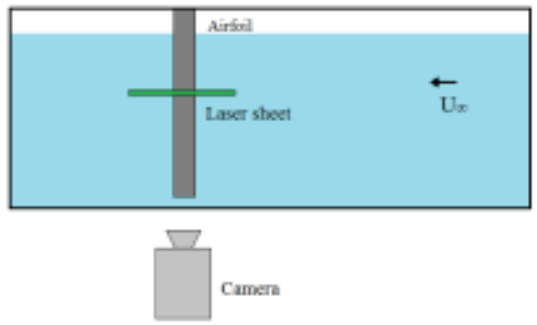

Figure 3. Side view of experimental setup

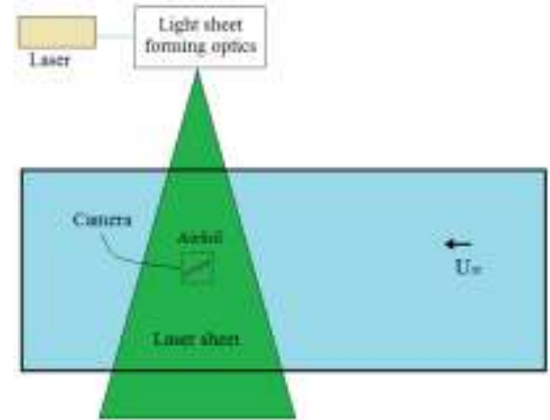

Figure 4. Top view of experimental setup 
In order to obtain a field of view that is at least 3 chords in streamwise direction and 2 chords in normal direction and accounting for a spatial resolution of at least $2.5 \mathrm{~mm}$, a magnification value of $\mathrm{M} \sim 14 \mathrm{pix} / \mathrm{mm}$ is chosen. For a particle displacement of around 3 pixels, and for the free-stream velocity of $66 \mathrm{~mm} / \mathrm{s}$, the time between exposures is fixed to $\Delta \mathrm{t}=0.003 \mathrm{~s}$. Special consideration is taken to prevent shadowing of pictures by the wing body. Note that the camera is adjusted depending on where the airfoil is located in a given phase in the cycle, to ensure it is contained in the frame with no shadowing.

The flow is seeded with Titanium Dioxide $\left(\mathrm{TiO}_{2}\right)$ particles of $3 \mu \mathrm{m}$ diameter, with particle density $\rho_{\mathrm{p}}=4.23 \mathrm{~g} / \mathrm{cc}$. The Stokes time is $\tau=\rho_{p} d_{p}^{2} / 18 \mu_{f}=2.12 \mu \mathrm{s}$, which is small enough for particles to follow the flow adequately and for a very small terminal velocity. The desired concentration is around 10 particles per interrogation window size, which is set to 32 × 32 pixels. This results in $\sim 3 \mathrm{~g}$ of $\mathrm{TiO}_{2}$ particles for $\sim 5000$ gallons of water. Around 10 drops of emulsifier are used to ensure particles are suspended in water for a longer period of time. The particles are mixed with a Sonicator, which breaks down clumps using high frequency vibrations. The channel is accelerated before adding the particles for better mixing. The mixture of particles and emulsifier is added at small increments to the water channel.

A control box is used to synchronize all the signals (lasers, camera, shutter, and motors) to a very high accuracy, on the order of microseconds. A frequency divider is used together with a time delay to ensure the pictures are taken at a very precise point in the motion. While the data is being acquired, two external channels monitor the motion of each motor, which is checked to ensure all components are synchronized as the experiment is run.

\section{B. Test cases}

The non-dimensional parameters to be explored in the current study are reduced frequency $(k)$ and reduced amplitude $\left(h_{0}\right)$, described below:

$$
k=\frac{\pi f c}{U_{\infty}} \quad h_{0}=\frac{h_{\max }}{c}
$$

The Strouhal number can be defined as a function of the two, described below:

$$
S t=\frac{2 f h}{U_{\infty}}=\frac{2 k h_{0}}{\pi}
$$

Four cases are selected, with reduced frequency ranging from $k=0.5$ to $k=1$, and reduced amplitude ranging from $h_{0}=0.25$ to $h_{0}=1$. These cases result in Strouhal numbers ranging from $S t=0.16$ to $S t=0.32$. Cases 1 through 4 plotted in $k-h_{0}$ space are shown in Figure 5.

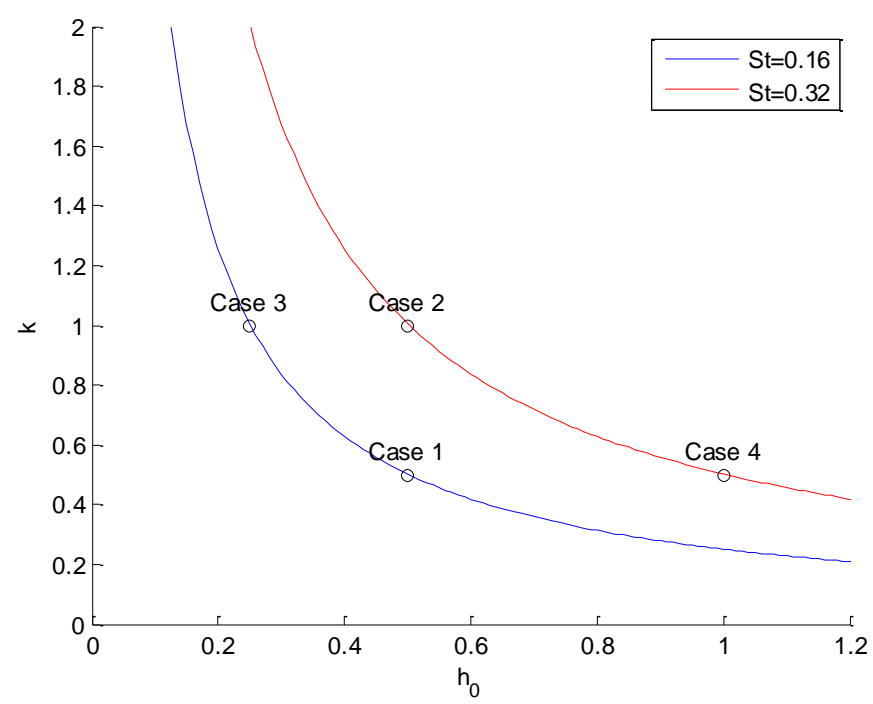

Figure 5. Cases 1-4 in $k$ - $h_{0}$ space 


\section{Kinematics}

This study focuses on a narrower range for the amplitude of the effective angle of attack, with $\alpha_{\text {eff,max }}=$ $5.65^{\circ}$. These cases are named A1-A4. In addition, Baik's cases will be reproduced, having $\alpha_{\mathrm{eff}, \max }=14^{\circ}$ (W1-W4), for confirmation purposes and comparison with the new cases. Note that for all cases, the mean angle is $\alpha_{0}=8^{\circ}$ and the pitch leads with $\phi=90^{\circ}$.

The effective angle of attack can be described by the equation:

$$
\alpha_{\text {eff }}=\alpha_{0}+\alpha_{\text {eff, } \max } e^{i(2 \pi f t-\phi)}=\alpha_{0}+\underbrace{\theta_{0} \cos (2 \pi f t+\phi)}_{\text {geometric pitch }}+\underbrace{\alpha_{p l, \max } \sin (2 \pi f t)}_{\text {plunge-induced } \alpha_{\text {eff }}}
$$

Since the phase lag is $90^{\circ}$, the geometric pitch can be rewritten as:

$$
\theta(t)=\theta_{0} \cos (2 \pi f t+\phi)=-\theta_{0} \sin (2 \pi f t)
$$

The plunge motion is not straight-forward to calculate, as it must induce a sinusoidal effective angle of attack. To achieve this, the plunge speed to free-stream velocity ratio has to satisfy:

$$
\arctan \left(-\frac{1 d h}{U_{\infty} d t}\right)=\alpha_{p l, \max } \sin (2 \pi f t)
$$

The plunge must then be solved by integrating equation 11, and setting the proper initial condition. We introduce a function $\mathrm{F}$ of the form:

$$
h(t)=h_{0} c F(t)
$$

After some algebraic manipulation, equations 11 and 12 can be combined into:

$$
F(t)=1-\int_{0}^{t} \frac{U_{\infty}}{h_{0} c} \tan \left(\alpha_{p l, \max } \sin \left(2 \pi f t^{\prime}\right)\right) d t^{\prime}
$$

Since the plunge is at a maximum at the beginning of the cycle and at the center of the motion after a quarter of the period (with maximum speed), $F(t)$ must satisfy:

$$
F(0)=1 \quad F(0.25 T)=0
$$

In order to generate the kinematic curves for each motion, a MATLAB script is used. After specifying initial values $\left(c, U_{\infty}, v, \alpha_{0}, \alpha_{\text {eff,max }}, \phi\right)$ and selecting the $k$ and $h_{0}$ for a given case, the script finds the Strouhal number (St), the frequency $(f)$, and the period $(T)$. It then finds the correct value of $\alpha_{p l, m a x}$ by iterating over values of it, until the following equation (obtained after variable substitution and algebraic manipulation of equation 13) is satisfied:

$$
S t=\frac{1}{\pi} \int_{0}^{\pi / 2} \tan \left(\alpha_{p l, \max } \sin (\zeta)\right) d \zeta
$$

The amplitude of the pitch motion $\left(\theta_{0}\right)$ can then be found using:

$$
\theta_{0}=\alpha_{p l, \max }-\alpha_{\mathrm{eff}, \max }
$$

Once $\theta_{0}$ is obtained, the geometrical pitch can be calculated with equation 10 . The plunge can be calculated directly by integrating equation 13 , and using equation 12 .

Cases A1-A4 will have greater pitch oscillation amplitude than cases W1-W4, in order to produce a narrower effective angle of attack range. A comparison of kinematic curves is shown in Figure 6. The smooth kinematic curves are filtered to reduce the total number of points, which allows the commands to be input in the motors. The filter removes points in each of the curves that are close to each other or that have similar slopes, reducing the total number of points to $\sim 65$. 


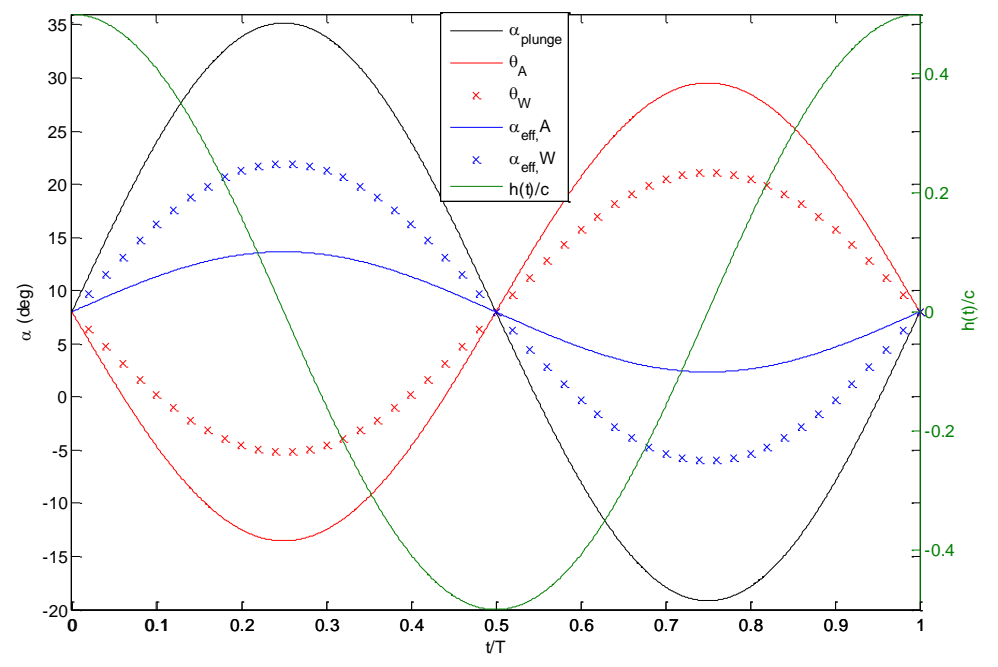

Figure 6. Kinematic curves comparison of $A$ and $W$ ranges, for Case 1

Note that in order to generate a smaller effective angle of attack amplitude, the shallow stall cases require a more aggressive pitch motion than the deep stall cases, since the plunge-induced angle of attack remains constant.

Table 1 summarizes the tests cases in $\mathrm{k}-\mathrm{h}_{0}$ space for this study, with relevant kinematic parameters. Note that most parameters are the same for the deep and shallow dynamic stall motions, with the exception of the pitch amplitude.

\begin{tabular}{|c|c|c|c|c|c|c|c|}
\hline Case & $\mathbf{S t}$ & $\mathbf{k}$ & $\mathbf{h}_{\mathbf{0}}$ & $\left.\boldsymbol{\alpha}_{\mathrm{pl,} \max } \mathbf{(}^{\mathbf{9}}\right)$ & $\left.\boldsymbol{\theta}_{\mathbf{0}, \mathbf{A}} \mathbf{(}^{\mathbf{}}\right)$ & $\left.\mathbf{\theta}_{\mathbf{0}, \mathbf{W}} \mathbf{(}^{\mathbf{}}\right)$ & $\mathbf{T}(\mathbf{s})$ \\
\hline 1 & 0.16 & 0.5 & 0.50 & 27.18 & 21.53 & 13.18 & 7.254 \\
2 & 0.32 & 1.0 & 0.50 & 47.77 & 42.11 & 33.76 & 3.627 \\
3 & 0.16 & 1.0 & 0.25 & 27.18 & 21.53 & 13.18 & 3.627 \\
4 & 0.32 & 0.5 & 1.00 & 47.76 & 42.11 & 33.76 & 7.254 \\
\hline
\end{tabular}

Table 1. Kinematic parameters for all cases

\section{Data Acquisition and Post-Processing}

For force data acquisition, tests are run in water and in air, to obtain the net hydrodynamic forces. For each case, 6 sets of 31 cycles are recorded. The data is then filtered using a Butterworth low-pass filter of order 10. The cut-off frequency set to $1.5 \mathrm{~Hz}$ to remove structural vibration and signal noise. Pre-trigger data is taken at the center of the channel, with the plate aligned in the flow direction $(h=0, \theta=0)$, which is then subtracted from results. For each set, the first 5 and the last cycle are discarded to remove start-up and stopping transienst. This results in 150 cycles of useful data per test case. The data is interpolated to get 200 points per cycle, and then an ensemble average is performed to obtain final force data results.

A statistical assessment of the processed force data yields a very narrow $95 \%$ confidence interval band for all test cases, due a small standard deviation and a large number of samples. Other tests are performed to ensure the force sensor does not drift significantly as the experiment is run for a long time, and to ensure the axial forces are negligible when the wing is at zero angle of attack in the center of the channel. These are not included in the present report for conciseness.

For PIV image acquisition, a set of 50 useful images is acquired for each test case, having discarded the first 5 in each set to remove transient effects. The images are post-processed by using a cross-correlation two-pass algorithm performed on a pair of images, which determines the particle displacement at selected grid points. A median filter is then applied which removes outliers in the velocity fields. Results for vorticity can be obtained with the final velocity flow fields. 


\section{Results and Discussion}

\section{A. Flow Topology and Development - Particle Image Velocimetry (PIV)}

Results for free-stream component of the velocity (u-velocity) and vorticity contours for the shallow dynamic stall (A cases) are shown in Figures 7-8, for 12 phases in the cycle. The plotted quantities are normalized by the free-stream velocity and the airfoil chord. In the vorticity plots, regions of low vorticity are omitted for clarification purposes. In addition, each figure has streamlines tangent to the flow, to observe if there are regions of recirculation present. The color axis is fixed to a set range for all plots for better comparison.

There are observable similarities in flow topology for all 4 cases. There is a high shear region emanating from the trailing edge throughout the cycle, particularly noticeable in the downstroke. Small LEVs are seen forming at the beginning of the downstroke, but they remain stationary close to the leading edge. They do not travel along the chord until around the middle of the cycle, at which the pitch rate is the highest. The LEV then advects quickly, traveling from LE to TE in just a few phases. In contrast to the $\mathrm{W}$ cases reported by Baik et al [1] which show the development of a LEV for all cases, in the present A cases the LEV is smaller.

As found in Baik et al [1], the reduced frequency is the dominant parameter in flow topology. For cases of high reduced frequency (2\&3), the LEV forms during the upstroke, and then it advects downstream in the second half of the cycle. Note that the location of the LEV with respect to the chord is almost the same for both cases, with the detachment occurring around $\mathrm{t} / \mathrm{T} \sim 0.75$. For cases of low reduced frequency $(1 \& 4)$, the LEV grows larger in size throughout the downstroke, and then it advects quickly around the motion midpoint. It is completely detached by $t / T \sim 0.667$. The vortex is allowed to grow and develop more due to the larger physical time between phases. The effect of the St number on the flow topology is less pronounced, but still noticeable. Cases with higher St number show larger values of u-velocity than their low St counterpart, and the shear layers are more pronounced. The curvature of the streamlines is larger. The vorticity plots also confirm this trend; higher $S t$ cases produce higher values of vorticity around the leading edge and along the shear layer emanating from the trailing edge. The velocity and vorticity magnitude differences will yield larger forces on the airfoil, as analyzed next.

It is important to notice that though LEVs are present, they are small in size and their presence is significant only in a few phases. There are no regions of notable recirculation, as seen in the vorticity plots. Thus, the dominant effects for force generation are due to non-circulatory or pitch-rate effects. 


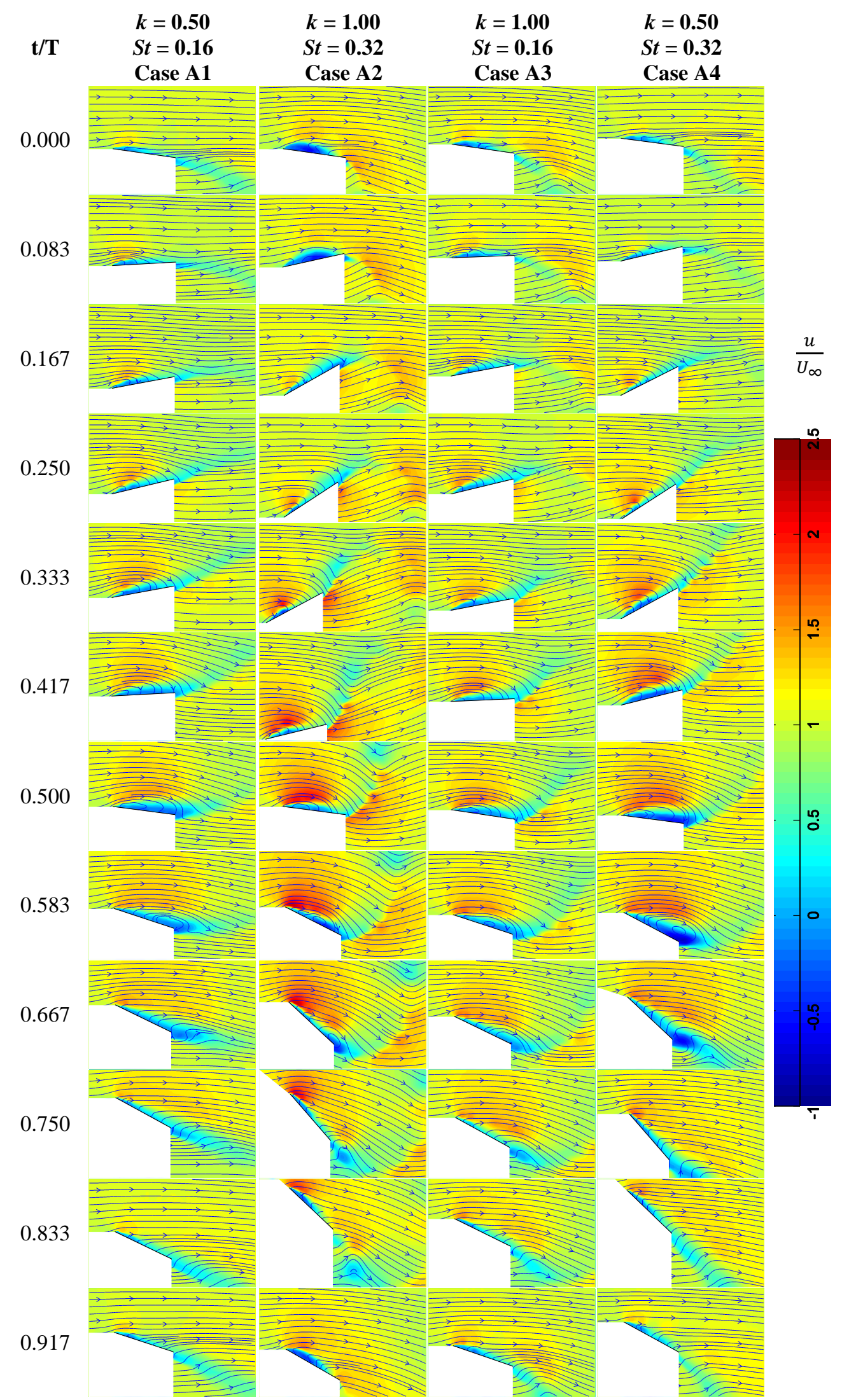

Figure 7. Normalized u-velocity contour plots for shallow stall cases (A1-A4) 


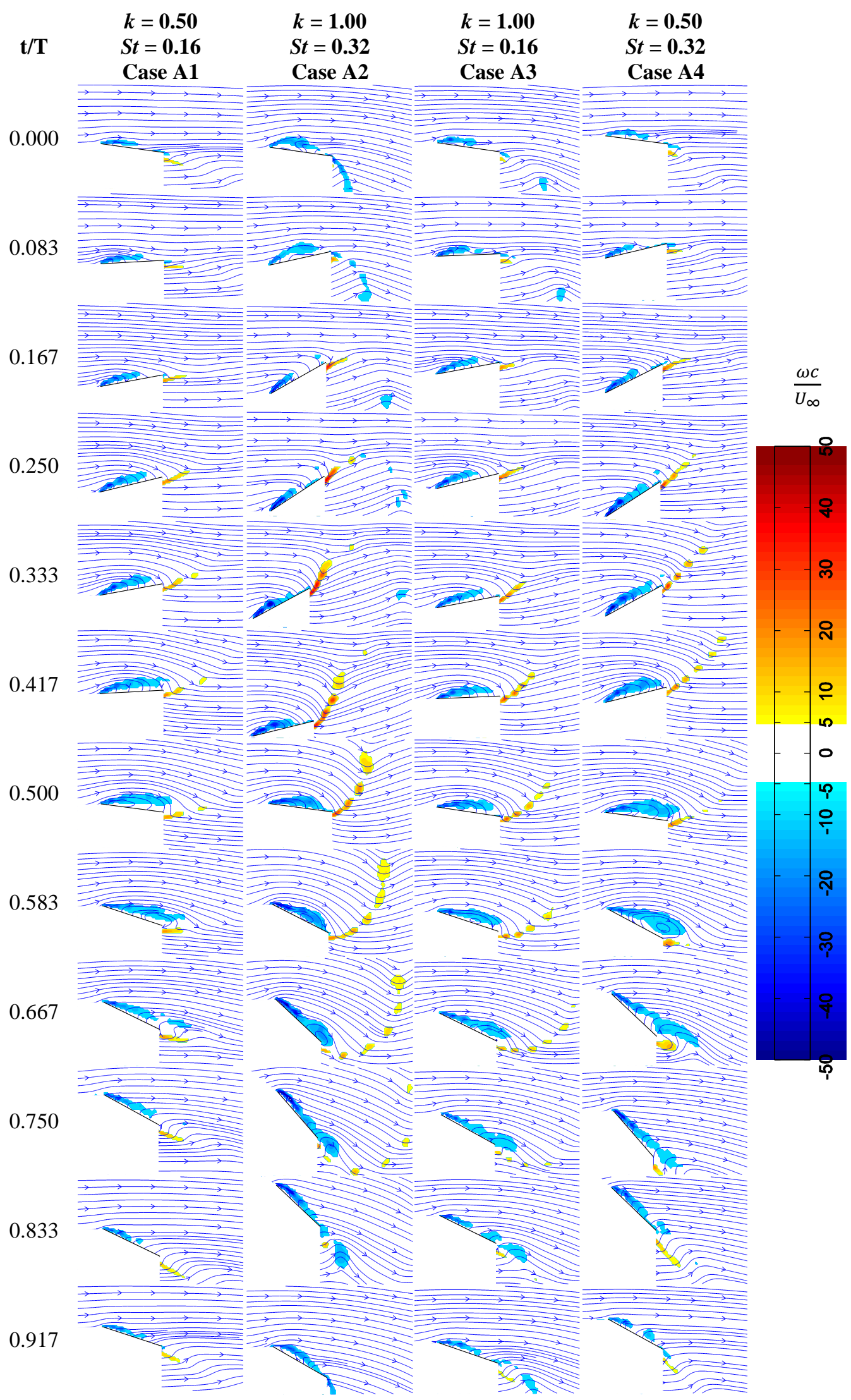

Figure 8. Normalized vorticity contour plots for shallow stall cases (A1-A4) 


\section{B. Axial and Normal Forces}

The net force acting on the flat plate is obtained by subtracting measurements in air from measurements in water. Results are shown in Figures 9 and 10. It is found that the axial forces $\left(\mathrm{F}_{\mathrm{x}}\right)$ are very small for both the deep and shallow stall cases, in a range barely within the limits of the force sensor resolution $(1 / 50 \mathrm{~N})$. The normal forces $\left(F_{y}\right)$ curves are smoother due to the larger magnitudes of force read by the sensor. As expected, cases with the same Strouhal number tend to produce similar normal forces, more noticeable in the deep stall cases.

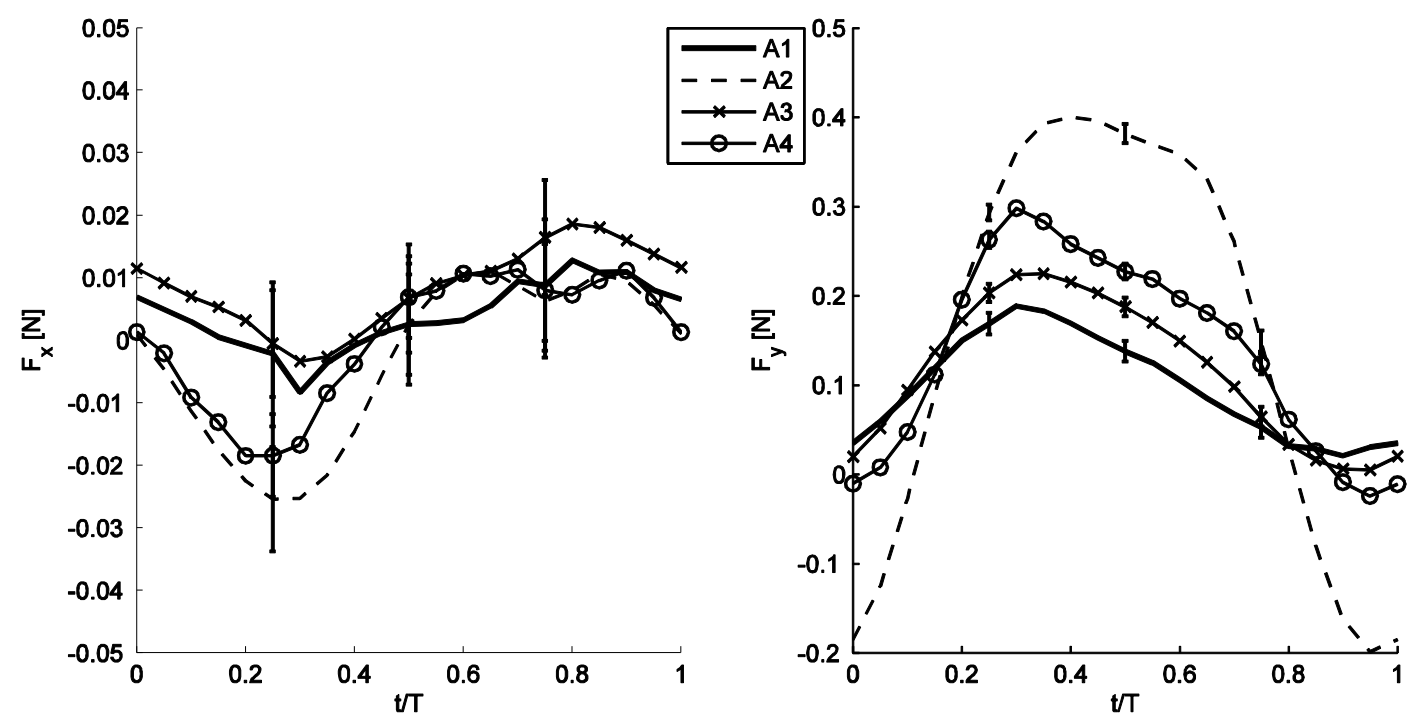

Figure 9. Net hydrodynamic forces for shallow dynamic stall (A cases)

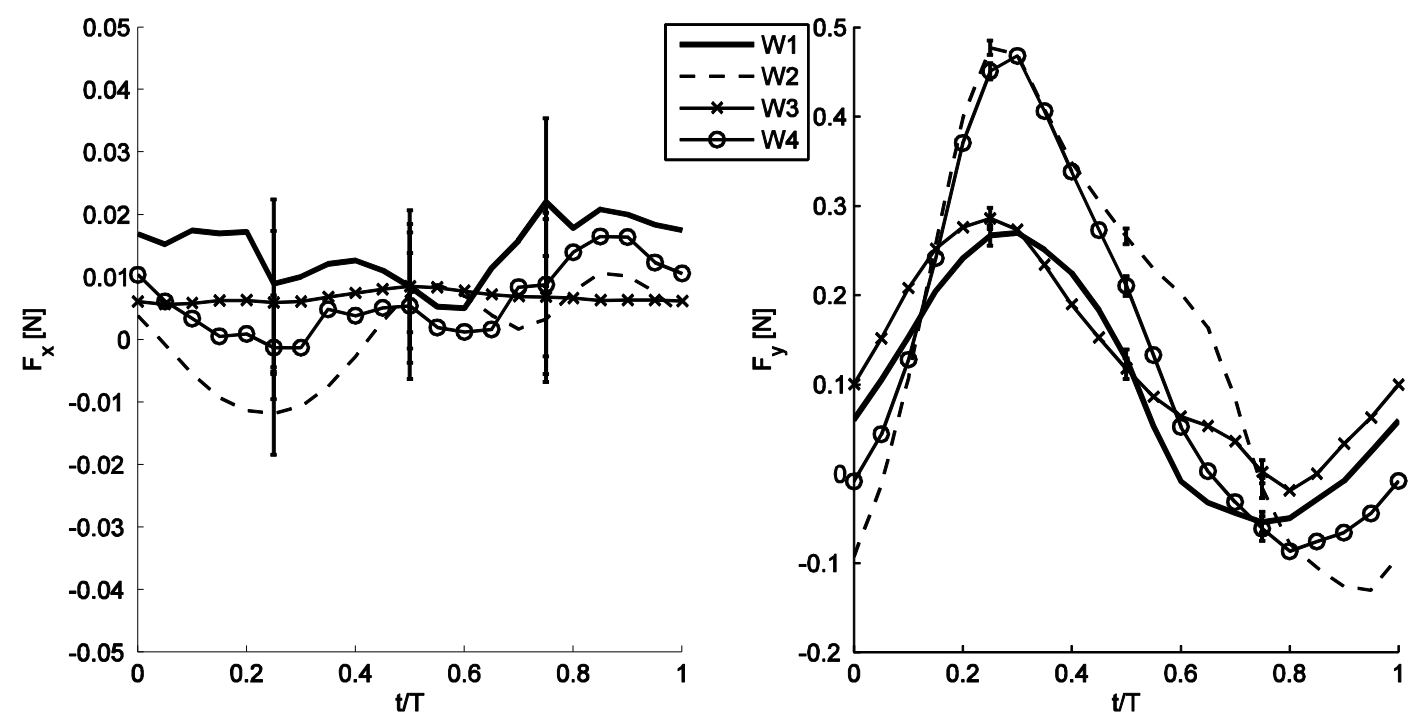

Figure 10. Net hydrodynamic forces for deep dynamic stall (W cases) 


\section{Lift and Drag Coefficients}

A comparison between lift and drag coefficients for shallow dynamic stall cases (A) and deep dynamic stall cases (W) is presented. As seen in Figure 11, the results are in agreement with Baik's work; cases with higher $S t$ number (1\&3) generate more lift than lower St number ones (2\&4), for both $\mathrm{W}$ and A ranges. The lift coefficient in A cases reaches a maximum value later in the cycle than for $\mathrm{W}$ cases; this difference is most noticeable for case 2 , where the A range peak occurs around $t / T \approx 0.50$, whereas for cases in the $\mathrm{W}$ range, it occurs around $t / T \approx 0.25$. For lower $S t$ number cases ( $1 \& 3$ ) in the A range, it occurs around $t / T \approx 0.30$, and for respective $\mathrm{W}$ cases it happens around $t / T \approx 0.25$. In addition, cases $\mathrm{A} 1, \mathrm{~A} 3$, and $\mathrm{A} 4$ have narrower ranges than respective $\mathrm{W}$ cases, and $\mathrm{A} 2$ has a wider one, though the maximum value is comparable. The minimum lift is also delayed in all shallow stall cases, happening around the end of the cycle and not around the three-quarter point in the cycle, as seen in deep stall.
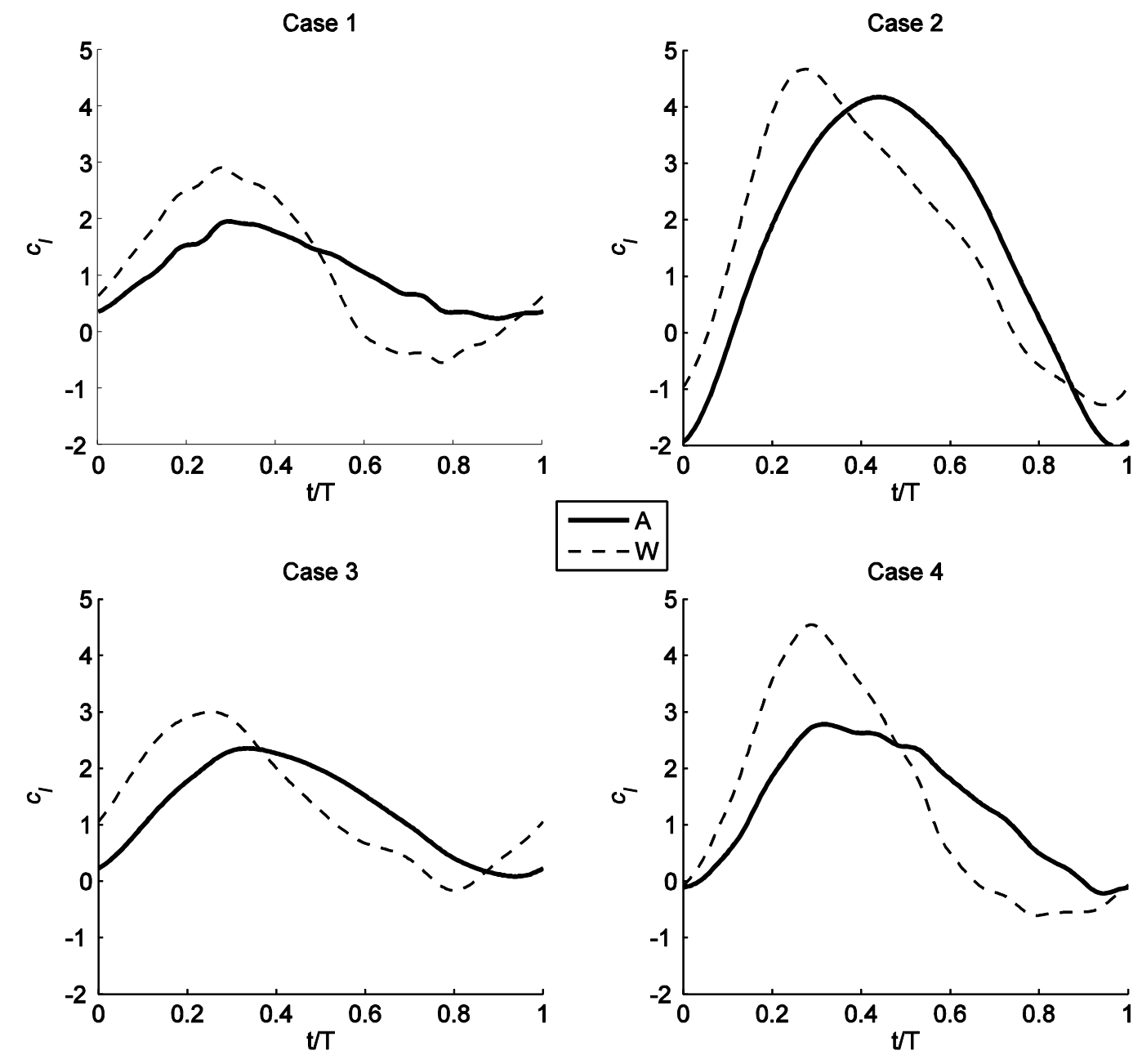

Case 4

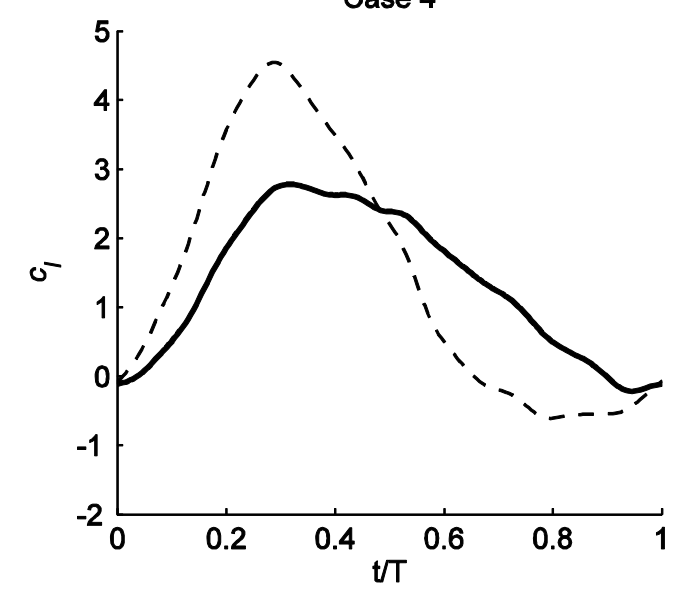

Figure 11. Lift Coefficients vs. Phase (t/T), for shallow (A) \& deep (W) dynamic stall

Drag coefficient plots, shown in Figure 12, also confirm previous results; cases with higher St number create larger amplitudes of oscillation in the drag forces, reaching higher thrust values in the first half of the cycle and lower ones during the upstroke. The main difference between the ranges is that the A cases have a higher drag than the $\mathrm{W}$ cases in the upstroke region of the cycle, where $t / T>0.5$. For the first half of the motion, the forces are comparable, reaching similar values of highest thrust. 
Case 1

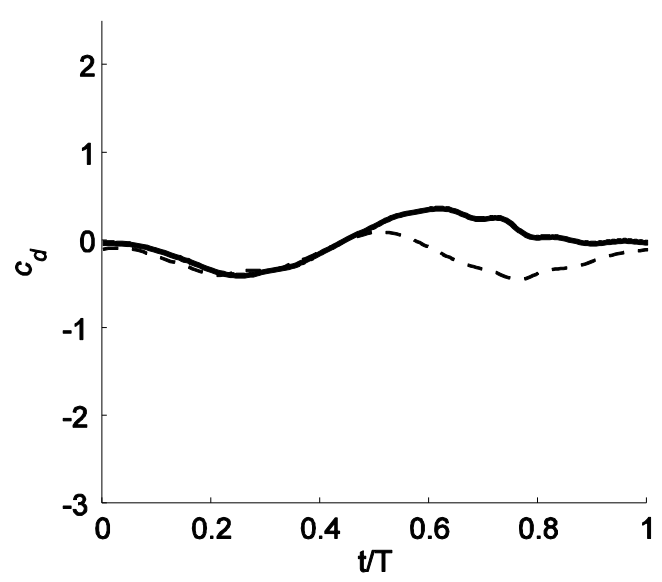

Case 3

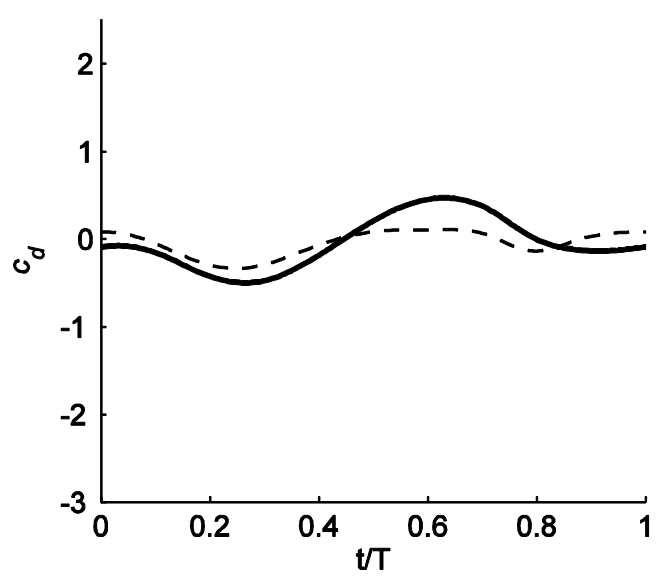

Case 2

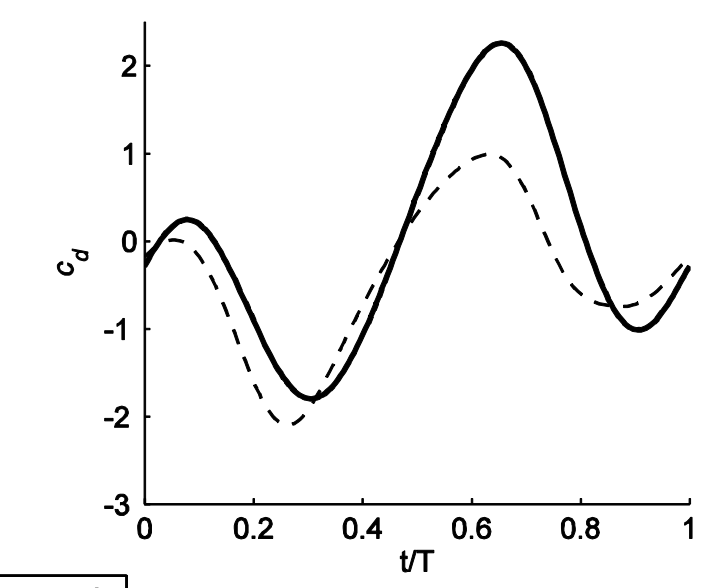

Case 4

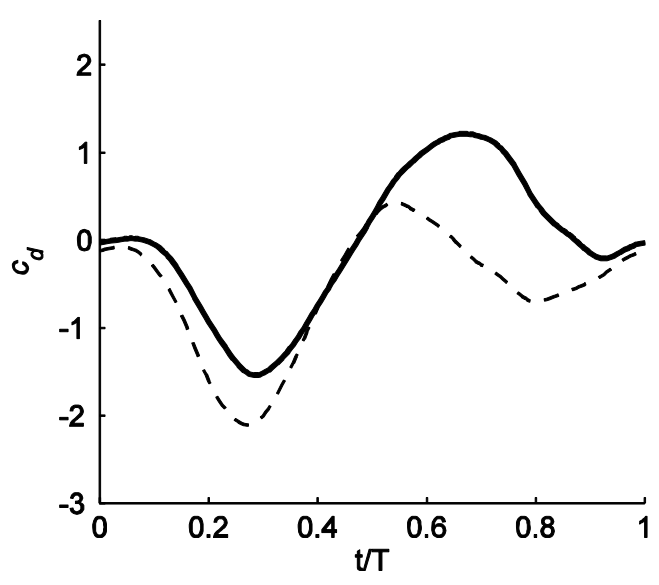

Figure 12. Drag Coefficients vs. Phase (t/T), for shallow (A) \& deep (W) dynamic stall

An interesting feature that these plots illustrate is that when the oscillation amplitude of the effective angle of attack is reduced, the net lift forces does not increase or decrease drastically, retaining comparable magnitudes for the most aggressive test cases (A2 \& W2).

Figures 13-14 show the lift and drag coefficients for each set of shallow and deep dynamic stall cases, with a comparison of classical aerodynamic lift given by $2 \pi \alpha_{\text {eff. }}$. As previously stated, there is a strong dependence on $S t$ number for both lift and drag forces. Cases of high $S t$ number $(2 \& 4)$ produce higher lift \& thrust. In addition, for cases with the same $S t$ number, a higher reduced frequency $k$ produces higher values of lift and thrust. The classical aerodynamic theory does not fare well with the shallow dynamic cases, even for ones with low $k$ and $S t$, whereas deep dynamic stall cases show better agreement. The slope of the curves in the middle region is similar to linear theory in both A and W cases, except for case A2, which shows a much higher lift and a very steep slope. It is interesting to note that around $\mathrm{t} / \mathrm{T} \sim 0.75$, all A curves meet around a lift coefficient value of $c_{l} \sim 0.5$, and all W curves do so around a value of $c_{l} \sim-0.5$. The drag curves show a transition from thrust generation in the downstroke to drag generation in the upstroke. 

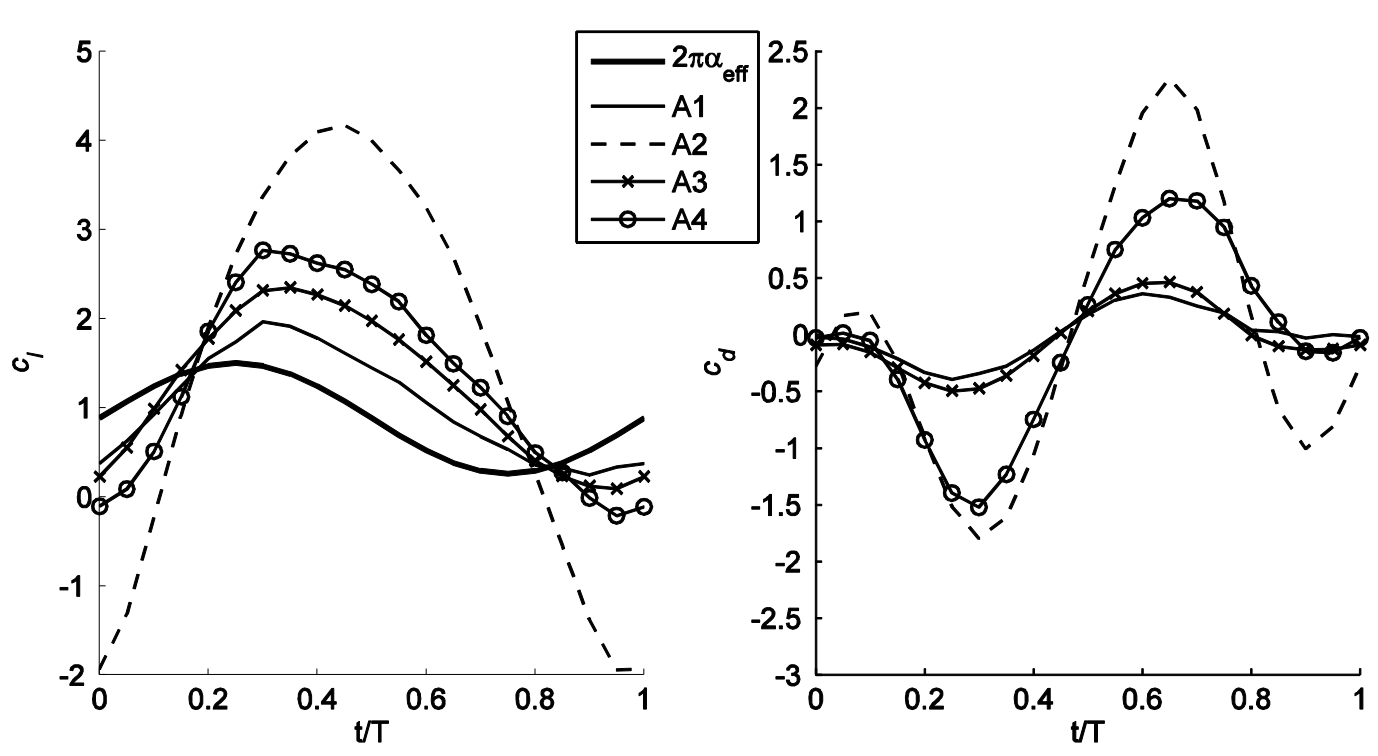

Figure 13. Lift and drag coefficients for shallow dynamic stall cases (A1-A4), with $2 \pi \alpha_{\text {eff }}$ comparison
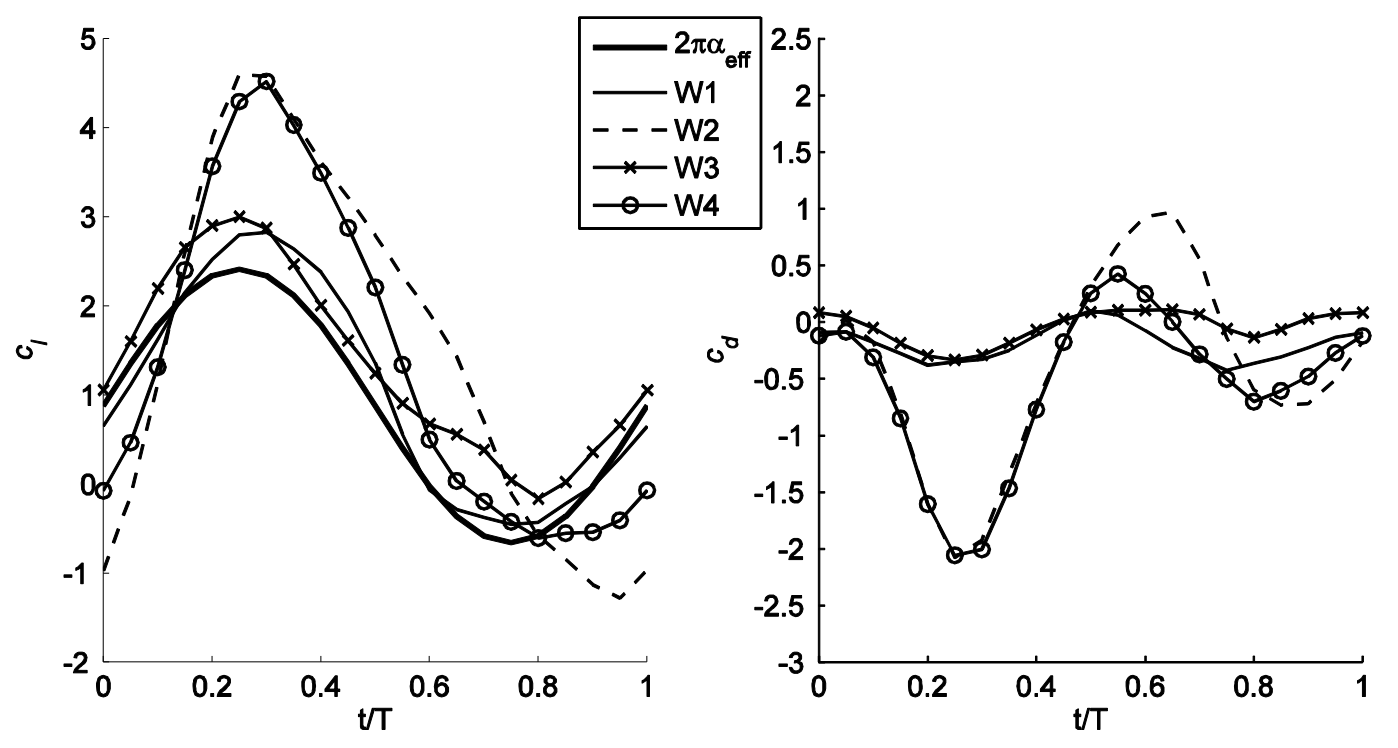

Figure 14. Lift and drag coefficients for deep dynamic stall cases (W1-W4), with $2 \pi \alpha_{\text {eff }}$ comparison

\section{Comparison to linear theory (Theodorsen \& Garrick)}

Figures $15 \& 16$ show the comparison between our experimental results and the linearized potential flow theory developed by Theodorsen ${ }^{19}$ and extended by Garrick ${ }^{20}$. The plots highlight the differences already pointed out - more circular lift coefficient distribution, narrower range in lift for cases 1, 3, and 4, and higher drag in second half of A cases. It can also be seen from these plots that the maximum lift case A2 does not always occur at the highest value of effective angle of attack, like in all other cases. It has a peak value around somewhere around $\alpha_{\mathrm{eff}} \approx 7.5^{\circ}$, corresponding to the bottom of the plunge $(t / T \approx 0.5)$. The minimum value also occurs later in the cycle, almost around the end of the upstroke. Drag coefficient plots show similar trends in both A and W cases: for all plots, the highest value for the thrust was achieved around the maximum value of $\alpha_{\text {eff }}$.

For both the deep and shallow dynamic stall, the low Strouhal number cases $(1 \& 3)$ tend to approximate the linear theory more closely than the high Strouhal number cases $(2 \& 4)$. The discrepancy is especially pronounced in the shallow stall cases. This is primarily due to the large geometric pitch that the A cases have; in order to offset the 
plunge-induced angle of attack, the airfoil has to rotate more aggressively and achieve larger amplitudes, which severely deviates from linear theory.

The lift coefficient in the A cases does approximate the linearized results for low $S t$ numbers (Cases A1 \& A3). The distribution is more circular,, and the areas contained in the curve are of similar size. For high St cases, (A2 \& A4), the linearized approximation fails to predict the measured forces significantly; the results are not slanted-oval-shaped, but more vertical. This difference is shown by the displacement of highest lift to the half-point in the cycle, as opposed to the quarter-point that linearized theory predicts. Similarly, the point of lowest lift happens around the end of the motion, instead of the three-quarter-point in the cycle.

The shallow dynamic stall cases show a similar trend; cases of low St tend to match linearized theory more closely than cases of high $S t$.
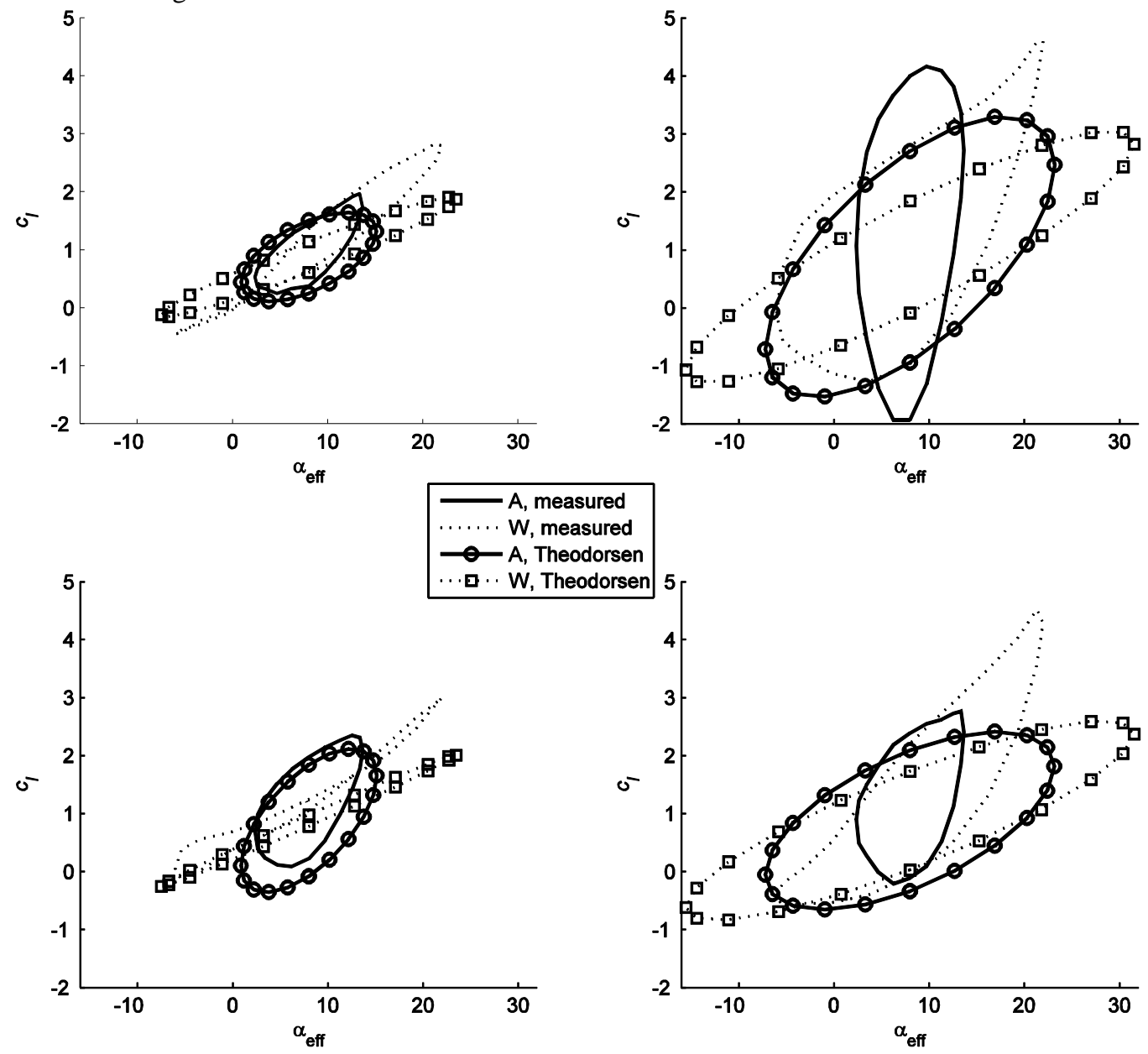

Figure 15. Lift coefficients vs. effective angle of attack of shallow (A) and deep (W) dynamic stall cases, with comparison to Theodorsen \& Garrick's linear theory 

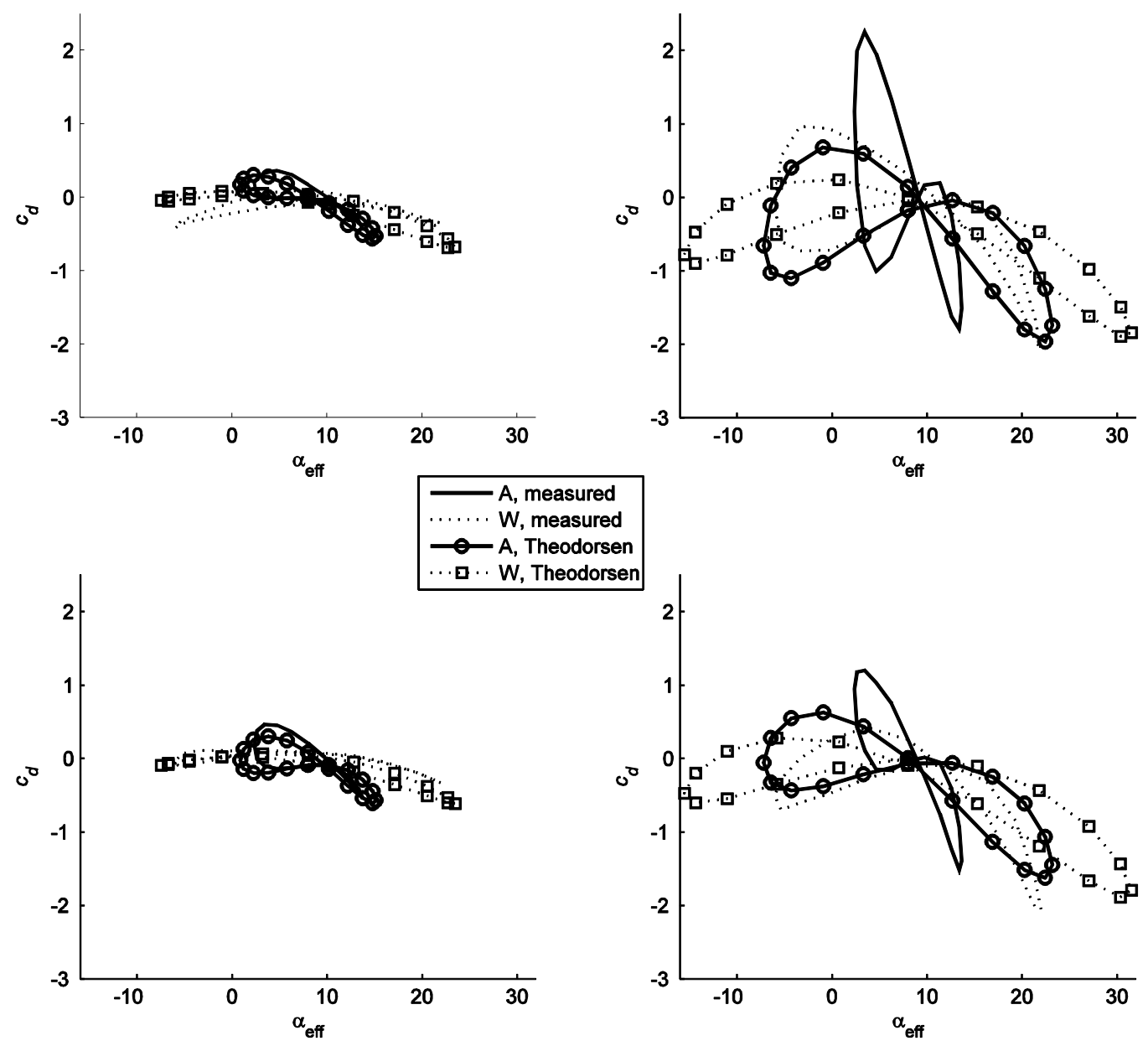

Figure 16. Drag coefficients vs. effective angle of attack of shallow (A) and deep (W) dynamic stall cases, with comparison to Theodorsen \& Garrick's linear theory

Table 2 displays the mean values for lift and drag coefficients of all cases from measured data, and Table 3 displays the mean values from Thedorsen's model. For the experimental work, the shallow stall cases produce a comparable amount of mean lift, differing by less than $8 \%$ in the most different case (A3 \& W3). For the drag, deep stall cases produce more thrust than shallow stall cases, and the difference is more pronounced when the $S t$ number is higher. In both shallow and deep stall cases with the same $S t$ number $(1 \& 3,2 \& 4)$, higher reduced frequency $k$ produces higher mean lift coefficient. This is consistent with flow topology findings, in which higher $k$ allow the LEV stay longer on top of the airfoil, which sustains lift. The trend is also seen for mean drag values in the deep stall cases, in which a higher reduced frequency yields more drag. Theodorsen's model underpredicts the lift for all cases, and it overpredicts the thrust for all cases except for case W1. The discrepancies between A \& W cases is small for lift coefficients, but Garrick's drag prediction shows significant discrepancies for cases of low reduced frequency. 


\begin{tabular}{|c|c|c|c|c|}
\hline & \multicolumn{2}{|c|}{$\boldsymbol{c}_{\boldsymbol{l}}$} & \multicolumn{2}{c|}{$\boldsymbol{c}_{\boldsymbol{d}}$} \\
\hline Case & $\mathbf{A}$ & $\mathbf{W}$ & $\mathbf{A}$ & $\mathbf{W}$ \\
\hline 1 & $1.037 \pm 0.579$ & $1.047 \pm 1.188$ & $-0.009 \pm 0.224$ & $-0.198 \pm 0.149$ \\
2 & $1.504 \pm 2.118$ & $1.578 \pm 1.992$ & $-0.024 \pm 1.196$ & $-0.412 \pm 0.854$ \\
3 & $1.249 \pm 0.789$ & $1.350 \pm 1.027$ & $-0.045 \pm 0.292$ & $-0.047 \pm 0.142$ \\
4 & $1.345 \pm 1.033$ & $1.404 \pm 1.792$ & $-0.047 \pm 0.805$ & $-0.566 \pm 0.698$ \\
\hline
\end{tabular}

Table 2. Mean force coefficients for shallow (A) \& deep (W) dynamic stall cases, from experimental data

\begin{tabular}{|c|c|c|c|c|}
\hline & \multicolumn{2}{|c|}{$\boldsymbol{c}_{\boldsymbol{l}}$} & \multicolumn{2}{c|}{$\boldsymbol{c}_{\boldsymbol{d}}$} \\
\hline Case & $\mathbf{A}$ & $\mathbf{W}$ & $\mathbf{A}$ & $\mathbf{W}$ \\
\hline 1 & $0.871 \pm 0.542$ & $0.875 \pm 0.725$ & $-0.092 \pm 0.269$ & $-0.186 \pm 0.262$ \\
2 & $0.859 \pm 1.713$ & $0.868 \pm 1.532$ & $-0.605 \pm 0.756$ & $-0.664 \pm 0.640$ \\
3 & $0.868 \pm 0.875$ & $0.876 \pm 0.800$ & $-0.150 \pm 0.261$ & $-0.179 \pm 0.225$ \\
4 & $0.865 \pm 1.086$ & $0.869 \pm 1.213$ & $-0.392 \pm 0.653$ & $-0.588 \pm 0.657$ \\
\hline
\end{tabular}

Table 3. Mean force coefficients for shallow (A) \& deep (W) dynamic stall cases, from Theodorsen's model

\section{Summary}

The influence of reducing the effective angle of attack oscillation amplitude on force generation of pitching and plunging flat plate airfoils with kinematics producing a sinusoidal effective angle of attack has been studied. Test cases are chosen over a range of reduced frequencies $(k)$ and reduced plunge amplitudes $\left(h_{0}\right)$, whose combination produce different Strouhal numbers $(S t)$. Results are compared with previous findings that used a wider range for $\alpha_{\text {eff,max }}$. The maximum lift coefficient occurs later in phase for $\mathrm{A}$ cases than for $\mathrm{W}$ cases, as well as the minimum. The lift coefficient ranges in A are narrower, except for Case A2, whose maximum value is comparable to W2. All A cases display larger drag for the upstroke as compared to W cases. Lastly, all 8 cases show maximum thrust happening around the maximum value of effective angle of attack.

PIV results show that the flow topology for cases of shallow stall is similar when the effective angle of attack is fixed. The reduced frequency is the dominant parameter in the formation and advection of LEVs. For cases with low $k$, the LEV that advects from leading to trailing edge is of noticeably larger size, and it travels faster in phase downstream. The $S t$ number dictates the magnitude of the u-velocity and vorticity, which leads to higher forces for higher $S t$ values. The presence of a shear layer is very pronounced for all shallow stall cases. No regions of high vorticity or recirculation zones are noticed, which suggests that the force generation mechanism is mainly due to non-circulatory and or rotation rate effects. 


\section{References}

[1] Baik, Y., Bernal, L. P., Ol, M. V., \& Shyy, W. (2011). Unsteady Force Generation and Vortex Dynamics of Pitching and Plunging Flat Plates at Low Reynolds Number. 49th AIAA Aerospace Sciences Meeting including the New Horizons Forum and Aerospace Exposition. Orlando, FL. AIAA 2011-220.

[2] Baik, Y., Rausch, J., Bernal, L. P., Ol, M. V., \& Shyy, W. (2010). Experimental Study of Governing Parameters in Pitching and Plunging Airfoil at Low Reynolds Number. 48th AIAA Aerospace Sciences Meeting including the New Horizons Forum and Aerospace Exposition. Orlando, FL. AIAA 2010-388.

[3] Baik, Y., Rausch, J., Bernal, L. P., \& Ol, M. (2009). Experimental Investigation of Pitching and Plunging Airfoils at Reynolds Number between $1 \times 10^{\wedge} 4$ and 6x10^4. 39th AIAA Fluid Dynamics Conference. San Antonio, TX. AIAA 2009-4030.

[4] Kang, C., Baik, Y., Bernal, L. P., Ol, M., \& Shyy, W. (2009). Fluid Dynamics of Pitching and Plunging Airfoils of Reynolds Number between $1 \times 10^{\wedge} 4$ and $6 \times 10^{\wedge} 4$. 47th AIAA Aerospace Sciences Meeting including the New Horizons Forum and Aerospace Exposition. Orlando, FL. AIAA 2009-536

[5] Ol, M. V., Bernal, L. P., Kang, C., \& Shyy, W. (2009). Shallow and deep dynamic stall for flapping low Reynolds number airfoils. Experiments in Fluids, 883-901.

[6] Taylor, G. K., Nudds, R. L., \& Thomas, A. L. (2003). Flying and swimming animals cruise at a Strouhal number tuned for high power efficiency. Nature, 707-711.

[7] Anderson, J. M., Streitlien, K., Barrett, D. S., \& Triantafyllou, M. S. (1998). Oscillating foils of high propulsive efficiency. J. Fluid Mech. , 41-72.

[8] Shyy, W., Lian, Y., Tang, J., Viieru, D., \& Liu, H. (2008). Aerodynamics of Low Reynolds Number Flyers. Cambridge University Press.

[9] Muller, T. J. (2001). Fixed and flapping wing aerodynamics for micro air vehicle applications. AIAA Prog Astronaut Aeronaut, Vol. 195.

[10] Freymuth, P. (1988). Propulsive Vortical Signatures of Plunging and Pitching Airfoils. AIAA J. , 881-883.

[11] Koochesfahani, M. (1989). Vortical Patterns in the Wake of an Oscillating Airfoil. AIAA J. , 1200-1205.

[12] Lai, J. C., \& Platzer, M. F. (1999). Jet Characteristics of a Plunging Airfoil. AIAA J. , 1529-1537.

[13] Shyy, W., and Liu, H. (2007). Flapping wings and aerodynamic lift: the role of leading-edge vortices. AIAA J., Vol. 45 , pp. $2817-2819$

[14] Dickinson, M. H., \& Gotz, K. G. (1993). Unsteady aerodynamic performance on model wings at low Reynolds numbers. J. Exp. Biol. , 45-64.

[15] Ellington, C. P., van den Berg, C., Willmott, A. P., and Thomas, A. L. R. (1996). Leading-edge vortices in insect flight. Nature, Vol. 384, pp. 626-630.

[16] Hover, F.S., Haugsdal, O., and Triantafyllou, M. S. (2004). Effect of angle of attack profiles in flapping foil propulsion. J. Fluid Struct, Vol. 19, pp. 37-47.

[17] Read, D. A., Hover, F.S., and Triantafyllou, M. S. (2003). Forces on oscillating foils for propulsion and maneuvering. J. Fluid Struct, Vol. 17, pp. 163-183.

[18] Schouveiler, L., Hover F. S., and Triantafyllou, M. S. (2005). Performance of flapping foil propulsion, J. Fluid Struct, Vol. 20, pp. 949-959.

[19] Theodorsen, T. (1935). General theory of aerodynamic instability and the mechanism of flutter.Tech. Rep. 496. NACA Report.

[20] Garrick, I. E. (1936). Propulsion of a flapping and oscillating airfoil. Tech. Rep. 567. NACA Report. 